\title{
ON THE ALLEN ENGINE AND GOVERNOR.
}

By Mr. charles T. PORTER, of Manchester.

The objects to be aimed st in carrying ont expansion in a steam engine, so as to effect the greatest economy, may be stated to be:that the full boiler-pressure should be carried into the cylinder at the commencement of tho stroke, and maintained up to the point of cut-off; - that the cut-off should be sharp, without reduction of the steam pressure by wire-drawing; - and that the exhaust should be invariable, allowing the steam pressure to act to the end of the stroke with all degrees of expansion, and discharging the steam with the least loss from back pressure during the return stroke.

The attainment of these objects has generally been considered practicable only by means of some kind of liberating valve-gear, in which the valve is released from the gear when wide open, and is closed suddenly by a spring or by the action of gravity; so as to avoid the reduction of pressure by wire-drawing, which ordinarily takes place thrơugh the gradual closing of the steam port by a slidevalve worked with a continuous motion. Many ingenious constructions of liberating valve-gear have been invented for effecting this object, more particularly in America, where the subject has received great attention; and amongst them may be specially mentioned the Corliss engine now introduced into this country, which has been found to accomplish the desired end very successfully.

In the Allen Engine however, forming the subject of the present paper, the preceding requirements are met by a direct continuous action of the slide-valves; which gives a sharp cut-off to the steam admitted at fill boiler-pressure, and a high range of expansion, together with a very free exkaust. At the same time this arrangement obviates the objection attending the principle of a liberating 
valve-gear, namely that the speed at which the engine can be worked is limited by the circumstance of the valve having to be disconnected from the driving gear and connected again at each stroke of the engine. The Allen engine admits of being worked at a very high speed, much higher than is usual in stationary engines ; and it maintains complete steadiness of motion at this high speed, combined with a great uniformity in the driving power throughout each revolution, although the steam is admitted at an unusually high pressure at the commencement of the stroke.

The engine is represented in Figs. 1 and 2, Plate 9, which show a side elevation and plan of an engine with cylinder of 12 inches diameter and 24 inches stroke. This engine has been running some months at the works of the Whitworth Co., Manchester, at a constant speed of 200 revolutions per minute or 800 feet per minute speed of piston, driving a considerable portion of the machinery in the works ; and a similar engine was worked at the late Paris Exhibition, running at the same speed. The engine is horizontal, fixed upon a bed-plate, and working an air-pump direct from the piston-rod, which is prolonged through the outer end of the cylinder. The slide-valves are worked by a link motion, which is controlled entirely by the governor, giving a variable degree of expansion according to the amount of work to be done by the engine; but the steam is always admitted to the cylinder at full boiler-pressure, without passing through a throttle-valve.

Valves and Valve Motion.-The steam slide-valves are shown in the longitudinal and transverse sections of the cylinder, Figs. 3 and 4 , Plates 10 and 11. The steam valves are independent of the exhaust valves, and two separate valves are employed, one for each port. The two steam valves $\mathbf{E} \mathbf{E}$ are driven with separate motions, independent of each other, so as to effect a sharp cut-off at each end of the cylinder by the rapid motion of each valve at the point of closing the port; the motion of one valve being rapidly accelerated at the point of cutoff, at the same time that the other valve is greatly retarded. Fach steam valve consists merely of an open rectangular frame sliding between two parallel faces, which are fixed, so that the valve is in 
equilibrium and its motion is not affected by the pressure of tho steam. The outer face $\mathbf{F}$, against which the back of the valve slides, is a rigid plate bridging across the port and fixed down solid to the port face; and it is adjusted so as to allow the valve to slide freely, but with so good a fit as to be steam-tighs. The travel of the valve in opening extends beyond the two faces, so as to admit the steam to the port at four places simultaneously, as shown in the separate diagrams, Figs. 13 and 14, Plate 15, in which the length of the valve-rods and the distance between the valves are shortened for convenience in the diagram. In Fig. 13 the sterm is shut off from the nearer port; and in Fig. 14 the further steam valve is shown at the point of opening the port, just previous to the commencement of the stroke, the crank being at that moment in the position shown by the dotted line.

In order to maintain the proper working of these valves, the back plate F, Figs. 3 and 4, is made very rigid, so as to prevent any yielding under the pressure of the steam; and the entire plate with its side supports being surrounded by the steam is consequently exposed to the same expansion as the slide-valves. In order to provide the means of adjustment in case of any wear, a packing strip is inserted on each side between the back plate and its side supports; so that by reducing the thickness of these packing strips the two faces can be readily let together at any time to fit the slide-valve. The wear is found however to be so exceedingly slight, on account of the absence of pressure on the rubbing surfaces, that there is no probability of any adjustment being required oftener than once a year.

The exhaust valves $G \mathrm{G}$ are also two separate valves, as shown in Figs. 3 and 4, and in the diagram, Fig. 15; but they move together and are driven by the same valve-rod. These slide-valves are of the ordinary form, and work in separate chambers, between the steam valves and the cylinder; they travel beyond the port face, so as to open for the exhaus, at the two edges simultaneously. As the steam valves however ojen at four places simultaneously, the exhaust valves are made of nearly double the width, as shown in Figs. 4 and 6 , in order to give a corresponding area of opening; and 
the extent of their motion is such as to give an area of opening for release of the steam more than double the largest area for admission. These valves work in equilibrinm through most of their stroke.

Althongh there are thus four separate valves for the steam and exhaust, the motion of all of them is obtained from a single eccentric, which is fixed on the crank shaft in the same position as the crank and without any lead, as shown in the diagram, Fig. 16, Plate 16. The eccentric is shown in Figs. 10 to 12, Plate 14, and has a curved slot in one side of the strap, which acts as the expansion link; and it is guided in its motion by being connected to the upper end of the vibrating lever $H$ centred below. Two ralve-rods, $I$ and $J$, are connected to the same slide-block in the link, and attached at the other end to two separate bell-crank levers upon intermediate rocking shafts, as shown in the diagrams, Figs. 13 and 14. One of these rocking shafts is made tubular, with the second running through it, as shown at $\mathrm{K}$ in Figs. 11 and 12, so that the two shafts work independently of each other. The second arms of the two bell-crank levers are connected respectively to the two steam valves, Figs. 13 and 14. The slide-block in the expansion link is carried by two side links from the arm of the governor, as shown in Fig. 1, so that its position is regulated entirely by the governor, the block being lowered so as to cut off the steam earlier whenever the governor balls begin to fly out in consequence of any increase of velocity.

The motion of the exhaust valves is invariable, and they are connected direct to a point L, Figs. 10 and 12, beyond the outer end of the link slot. A greater travel is thereby given to the exhaust valves, as shown in Fig. 15, than the greatest travel of the steam valves; and this motion is continued the same during every change of expansion produced by shifting the slide-block from which the steam valves are moved, so that the exhaust continues equally free with every degree of expansion. The point of connection to the link is so placed as to give the required lead to the exhaust valves, and is arranged beforehand to suit the intended speed of piston. The exhaust valves are driven through an intermediate rocking shaft $M$, Fig. 12, for reversing the motion obtained from the eccentric; and a pair of ralve spindles are used, one at each odge of the two ralres, as 
shown in Figs. 4 and 6, and working within the intermediate exhaust port, Fig. 3. The spindles are guided at each end, and each valve is connected to them by two studs fixed in the valve, which fit into slots in the spindles, working clear within the opening of the ports, Figs. 3, 4, and 15 . In the case of the steam valves, as the two valves have independent motions, the spindle for driving the further valve passes free through a short tube in the lower side of the nearer valve, Fig. 4 ; the two spindles are made to clear each other by the valves being placed out of line with each other, as shown in the elevation of the steam port faces, Fig. 5 ; the spindles are kept central in each of the valves.

All the valves are readily accessible by simply removing the nuts of the steam-chest cover N, Figs. 3 and 4, as the steam-chest is not cast solid upon the cylinder, but is a separate rectangular frame $\mathrm{O} O$, fitted down with a scraped joint on both faces, and secured by the same through-bolts that fix the cover $N$. This construction has the important practical advantago that the steam-chest can be entirely remored, and all the port faces can be readily got at like plain outside surfaces.

In this valve motion the proportion between the throw of the eccentric and the length of eccentric-rod, or the distance from the centre of the eccentric to the centre of the link, is made the same as the proportion between the main crank and the connecting-rod, which in this engine is 1 to 6 , or the length of connecting-rod is 3 times the stroke. Consequently as the eccentric is set exactly to correspond in position with the crank, the angular vibration of the one compensates for that of the other, and an exactly correct valve-motion is obtained, giving the same results for each end of the cylinder. A considerable difference often exists in the indicator figures taken from the opposite ends of a cylinder, in consequence of the discrepancy between the motions of the connecting-rod and the eccentric-rod; and the difference in speed of piston cluring the first $1^{\circ}$ of rotation of the crank at the extreme opposite ends of the stroke amounts to 28 per cent. of the higher speed when the connecting-rod is 6 times the crank, 33 per cent. when 5 times, and 40 per cent. when 4 times the crank. 
In such cases therefore the point of cut-off is not the same in the two strokes of the piston; but in the present engine the two strokes are identical throughout, in consequence of the eccentric and connecting-rods having exactly parallel and simultaneous motions.

The connection of the expansion-link to the steam valves by the intermediate bell-crank levers introduces in effect a toggle-joint movement, which has the important advantage of allowing the length of the valves to be reduced very considerably, because more than half of the lap or useless motion of the valves after having covered the ports is dispensed with by their motion being greatly retarded at that time; whilst the opening for steam admission is correspondingly increased by the motion of the valves being accelerated at the opposite extremity of their travel. This variation in the motion is shown by the diagram of the link motion in Fig. 16, and also in Figs. 13 and 14, together with the varying angular motion of the bell-crank levers on the rocking shaft, the reference letters indicating corresponding positions throughout. The result is seen to be a very great range of variation in the motion of the valves, giving on the one hand a wider steam opening and a sharp cut-off with high degrees of expansion, and on the other hand a very slow motion of the valve during the time that it is simply retaining the port closed. The whole action is obtained with a continuous and perfectly smooth motion throughout.

In the adjustment of the centres of the link motion, the centre of the supporting lever H, Fig. 12, is slightly lowered, so that its upper end vibrates entirely below the centre line, as shown in Fig. 16, in order thereby to equalise the extent of tipping of the link at the twa extremities of its vibration. This gives a slightly increased lead to the steam-valve opening at the further end of the cylinder, where the motion of the piston is the more rapid on account of the smaller are of rotation of the crank; but the point of cut-off of the valves is exactly the same at the opposite ends of the cylinder for each degree of expansion, and the point of cut-off can be varied from 4-10ths of the stroke to the very commencement of the stroke.

Indicator diagrams are shown in Fig. 18, Plate 18, taken from this engine, at the speed of 200 revolutions per minute or 800 feet 
per minute speed of piston; and those in Figs. 19 and 20, Plates 19 and 20, are taken from two larger engines with 18 and 20 inch cylinders working at the specd of 600 feet per minute. This speed of 600 feet per minute is the one that has been adopted in practice for these engines; but the continued successful working of those running at 800 feet per minuto shows the higher speed to be quite practicable. The indicator diagrams in Fig. 19 are taken from an engine at Messrs. Pooley's Cotton Mill, Manchester, having a cylinder' 18 by 30 inches, and working at 120 revolutions per minute; those in Fig. 20 are from an engine at Messrs. Evan Leigh's works, Manchester, with 20 by 36 inch cylinder, and working at 100 revolutions per minute. In lig. 19 are shown the effects of wet steam in destroying the sharpness of cut-off, as the boiler was sending over a great deal of water with the steam; and in Fig. 20 a sharp cut-off is shown, as the engine was working with dry steam. The vacuum shown in Fig. 20 is deficient, becanse the condenser was not in good order. In Fig. 19 the lower straight dotted line A shows the vacuum in the condenser, and the upper straight dotted line $B$ the vacuum in the exhaust chamber at the time of taking the dotted indicator diagram.

The valve motion shown in the drawings is arranged for an engine running always in one direction, as in the case of driving shop machinery; but when a reversing action is required, the expansion-link slot is simply prolonged to an equal distance below the centre line, and exactly similar actions are then obtained in the opposite direction, in consequence of the position of the eccentric being exactly in correspondence with that of the crank.

For the purpose of obtaining the full benefit of expansion, it is requisite, in addition to having the full boiler-pressure in the cylinder and providing a sharp cut-off, that superheated steam shonld be used, in order not only to prevent water from being carried over into the cylinder with the steam, but also to prevent any loss arising from the freshly admitted steam becoming condensed in the cylinder by contact with the cylinder surface which has been cooled in the previous expansion. About $50^{\circ}$ Fahr. of superheating is found desirable; and this proves a more efficient mode than sterm- 
jacketting, because the superheated steam on admission into the cylinder supplies heat to the very surfaces that have been cooled by exposure to the low-pressure expanded steam.

Speed of Piston-One of the objects aimed at in this engine is to work at speeds considerably higher than those ordinarily used in any but locomotive engines. For the following reasons it is considered that the speed of piston should not be less than 600 feet per minute; but these engines have been worked continuously at the higher speed of 800 feet per minute with complete success, and it is believed that still higher speeds may in some cases be employed with advantage. The valves and the whole of the working parts are so well adapted to maintain a high speed, that the practical objections which ordinarily limit the speed of piston to lower rates do not apply in the case of the present engine.

The principal object in adopting the high speed of piston is to obtain a sufficient reciprocating force in the moving parts for balancing the initial force of the steam upon the piston when admitted at full boiler-pressure at the commencement of the stroke, so as to relieve the crank from strain on passing the centres; and also to equalise more fully the driving force upon the crank during the entire stroke. At the commencement of each stroke, an accelerating force is required sufficient to put in motion the mass of the reciprocating parts at the velocity at which the piston moves from a state of rest; and the speed of piston is adjusted so as to make this required force as great as the actual full force of the steam upon the piston when admitted at the boiler pressure, so that the two forces are in equilibrium at that point and the crank pin is thereby relieved from strain when passing the centre. This accelerating force imparted to the piston at the commencement of the stroke is given out again during the retardation of the piston in the latter half of the stroke, and thus compensates for the diminishing driving force of the expanding steam, and acts to equalise the driving power upon the crank. A similar action takes place in all engines, but at the speeds of piston ordinarily employed its extent is too small to produce any material effect; and it is only with a high speed that the effect becomes 
important, since the force recuired to put in motion or to stop the reciprocating parts increases as the square of their velocity of motion. Where a high speed is combined, as in this engine, with an unusual weight of the reciprocating parts and a short stroke, the inertia of these parts acts as a powerful reciprocating flywheel to equalise the driving power of the engine throughout the revolution of the crank.

In the present engine the weight of the reciprocating parts is $470 \mathrm{lbs}$, the cylinder 12 inches diameter by 24 inches stroke, and the number of revolutions 200 per minute, or one revolution in 0.3 second. Taking the motion of the piston in the first $1^{\circ}$ of revolution of the crank at the commencement of the stroke, the extent of motion will be the versed-sine of an angle of $1^{\circ}$ with a radius of 1 foot, or 0.000152 foot in the time of $\frac{1}{3} \frac{1}{6}$ of 0.3 second, or $\frac{1}{1200}$ second; which is equivalent, as regards the accelerating force required to produce it, to a motion of 219 feet in one second, the space passed through under a uniform accelerating force being in proportion to the square of the time. This motion of 219 feet in one second is 13.7 times the effect of gravity (16.08 feet in one second); and consequently the force required to impart the velocity amounts to 13.7 times the weight of the reciprocating parts ( $470 \mathrm{lbs}$.), making a total force of $6439 \mathrm{lbs}$, which is equal to a pressure of $57 \mathrm{lbs}$. per square inch upon the area of the 12 inch piston. It follows therefore that the steam at the full pressure of $57 \mathrm{lbs}$. may be admitted suddenly to the cylinder at the commencement of the stroke, without causing any strain upon the crank pin; and indeed this full pressure is absolutely required upon the piston at that moment, in order to prevent a strain in the opposite direction upon the crank pin from. the inertia of the reciprocating parts.

This principle is carried out in the regular working of the engine shown in the drawings, which has been running constantly for some months at the Whitworth Co.'s works with the speed of piston of 800 feet per minute; it is worked in direct communication with a boiler of $60 \mathrm{lbs}$. pressure, without any intermediate throttle-valve to reduce the pressure of the steam before entering the cylinder. The indicator figures given in Figs. 18, 19, and 20, show an excessive variation of pressure upon the piston throughout the stroke, ranging 
from $70 \mathrm{lbs}$. down to $10 \mathrm{lbs}$. per inch total pressure above a perfect vacuum; but in order to ascertain the effective driving power upon the crank, the whole amount of the pressure due to the inertia of the reciprocating parts has to be deducted at the beginning of the stroke, and the same amount has to be added to the indicator figure at the end of the stroke, both amounts being gradually reduced to nothing at the middle of the stroke. This correction acts directly to compensate for the inequality of the indicator figure, and the effective driving power throughout the stroke is thereby equalised to a remarkable extent. In the diagram, Fig. 17, Plate 17, the pressure produced upon the crank pin by the inertia of the reciprocating parts during the acceleration and retardation of the piston in each half of the stroke is represented by the dotted line $\mathrm{AA}$, the amount of the pressure at the beginning of the stroke being represented by the distance $\mathrm{AC}$ from the horizontal base line $\mathrm{CC}$; this diminishes to nothing at the middle of the stroke, and becomes an equal amount of pressure $\mathrm{AC}$ at the end of the stroke, but in the opposite direction. The rate of change is uniform, during equal spaces passed through by the piston, and is represented by the straight dotted line $A A$, if the varying angular motion of the connecting-rod be neglected; but when this is taken into account, the result is modified practically to the slightly curved line B B.

For carrying out a high degree of expansion a high speed of piston is essentially requisite, in order that a sufficient amount of equalising effect may be obtained from the inertia of the reciprocating parts, to compensate for the extreme variation in steam pressure which is consequent upon an early cut-off. Indeed in consideration of smoothness of running, the engine should be run so fast that the driving force produced by the highest pressure of steam cannot exceed the inertia of the reciprocating parts; and then a knock upon the centres becomes as impossible as it would be in a revolving sling.

A high speed of piston is also advantageous on account of the reduction in size of engine required to supply a given amount of 
power, whereby an important saving in space and cost is effected; and also on account of the increased uniformity of motion obtained with the higher speed. Taking the case of a single engine in place of a pair of coupled engines running at half the speed, the strokes are as frequent as those of the pair of engines, while the inequalities in the rotative force are not much larger, owing to the great equalising effects of the inertia of the reciprocating parts at the high speed. Moreover as the regulating power of the flywheel increases in proportion to the square of the number of revolutions in a given time, the same wheel has four times the regulating power when ran at double the speed.

The practical objections generally considered to apply to a high speed of working are, increased wear and tear, risk of hot bearings, cutting of the cylinders and pistons, and shaking loose in the fixings. But instead of any difficulties of this kind having been experienced, this engine runs smoothly and quietly, without tremor and without warming in the bearings, running continuously without requiring attention, and showing exceedingly slight wear in the cylinders, valves, and bearings. This result has been attained simply by good mechanical construction and workmanship; avoiding any unbalanced action, overhanging strains, insufficient stiffness of framing, inadequate bearing surface, or want of truth in workmanship. Unless all these conditions are carefully attended to, high speed is certainly not practicable; but when they are thoroughly carried out, no difficulty is experienced in working at any desired speed.

In the previous consideration of the effect produced by the inertia of the reciprocating parts, this has been taken as the same at each end of the stroke; but in reality a considerable difference is caused by the angular motion of the connecting-rod at the two ends of the stroke; and the actual motion of the piston during the first $1^{\circ}$ of rotation of the crank, instend of being 0.000152 foot at each end, is 0.000178 at the outer end of the stroke, and only 0.000127 at the inner end. Consequently, from this approximate mode of calculating by the difference in motion of the piston during the first degree of rotation from each end of the stroke, the pressure on the piston required to balance the inertia, instead of being $57 \mathrm{lbs}$. per inch at 
both ends as named before, would be $66 \mathrm{lbs}$. at the outer end and $47 \frac{1}{2} \mathrm{lbs}$. at the inner end. In the case of inverted vertical engines, the weight of the reciprocating parts acting vertically tends to equalise these amounts, by being added at the outer or upper end and deducted at the lower end. For this reason, and on account of the more correct support of the cylinder and the absence of overhanging strains, that form of engine seems preferable for the highest speeds.

Condenser and Air-Pump.--In the arrangement of the condenser and air-pump the object has been to meet the difficulty of combining the advantage of a simple direct-acting air-pump with the very unusually bigh speed of working, 200 revolutions per minute. This has been effected with complete success by the construction adopted; a vacunm of 27 inches of mercury is maintained with great steadiness, and the air-pump works quite quietly and without noise at the full speed, and keeps thoroughly in order withont requiring any attention.

The air-pump, as shown in Figs. 1 and 2 and in the transverse section, Fig. 7, Plate 12, is a nearly cubical box filled with water, with a plunger working through a stuffing-box in the lower part; the plnnger is attached to the pistori-rod and forms a continuation of it. The end of the plunger is made of a parabolic shape, as shown in Fig. 1, for displacing the water easily; and the plunger works entirely immersed in the water, simply displacing its own bulk of water at each stroke.

The inlet and outlet valves are all placed in the top plate of the box in two parallel rows, three inlet valves in one row and three outlet valves in the other, as shown in the plan, Fig. 2. They are india-rubber disc-valves with 8 inches diameter of opening, Fig. 7, and slide parallel upon their centre spindles without any bending of the india-rubber, being fitted with a metal plate and a long centre bush to guide the discs steadily in opening and closing, and to prevent any wear upon the edge of the india-rubber. The lift of the valves is about $\frac{1}{2}$ inch, and in order to obtain a quick action in closing they are closed by spiral springs, which load the valves to the extent of $\frac{l}{4} \mathrm{lb}$. per square inch. These spring's are found 
necessary upon the outlet valves as well as the inlet, in consequence of the quickness of the action required to close them 200 times per minute; and it was found that even at the speed of 120 times per minute there was a loss of $1 \mathrm{lb}$. per inch in the vacuum when the springs were not used, from the valves not closing promptly enough.

The condenser forms one half of the chamber above the air-pump, and the hot-well the other half, as shown in Fig. 7. The injection is introduced by a single opening in the centre of the condenser, with the full area of the pipe, in order to avoid any risk of the injection opening getting contracted by accumulation of deposit in a spreader or rose; and it has been found that no pereeptible difference is caused by this arrangement in the vacuum obtained with the injection. In consequence of the position of the valves in the top plate of the airpump chamber, the air entering from the condenser does not pass through the water, but simply passes over the surface of the water from the inlet to the outlet valves; and the water rising up to the outlet valves at each stroke ensures the discharge of the whole of the air. As no air gets to the lower portion of the air-pump chamber, the plunger works always in solid water, avoiding any churning action of mixed water and air; and indeed the effective piston of the air-pump may be considered to be, not the plunger, but the surface of the body of water that always remains in the air-pump chamber, which rises and falls at each stroke of the pump through a distance of less than one inch. The working velocity of the air-pump piston is therefore reduced in effect to only about 30 feet per minute, instead of 800 feet per minute the actral velocity of the plunger.

For the purpose of facilitating the passage of the air from the inlet to the outlet valves, the former are set at a small inclination below the horizontal position, as shown in Fig. 7, this idea having been suggested by a marine engine of Messrs. Maudslay and Field. The arrangement and position of this condenser and air-pump are very convenient for access to all the parts, the whole being above ground and at the level of the engine. It is held steady in its position by a connecting tie to the frame below and by the fixing of the exhaust-pipe above; and it serves as an additional guide for the smooth working of the piston-rod beyond the cylinder. The weight 
of the plunger moving at the full velocity of the piston serves also as an important addition to the compensating action of the inertia at the commencement and end of each stroke.

Governor.-The expansion gear of the engine is regulated entirely by the self-acting movement of the governor, Figs. 1 and 2 ; and for carrying this plan out satisfactorily it is essential to have a governor that is extremely sensitive to any change of velocity, and acts with great promptness upon the expansion gear, with power sufficient to shift it instantly to the full extent required. The governor used for the purpose has been designed by the writer as a modification of the ordinary Watt centrifugal governor, with the view of increasing its sensitiveness and quickness of action, and adding to the power available for overcoming the resistance of the valve motion. This resistance is however reduced to a very small amount in the present engine, on account of the valves being in equilibrium.

The governor is shown in Figs. 8 and 9, Plate 13, and consists of two revolving balls of small size, but moving at a high velocity, which pull up a heavy central weight, when they rise in consequence of an increased velocity of revolution; the balls are only about 2 to $3 \mathrm{lbs}$. weight, but the central weight is from 50 to $300 \mathrm{lbs}$. according to the size of the governor. The connection of the radius rods to the centre spindle is made with forked ends having considerable width of fork, as shown in Fig. 8, and fitting upon a pin which passes through the axis of rotation, Fig. 9. The friction which opposes the rise and fall of the balls is thus reduced, by the pressure upon the pin at the joints of the rods being diminished in consequence of their increased leverage; and the sensitiveness of the governor is thereby increased, its friction being much less than that of the ordinary governor.

With the very heavy revolving balls employed in the ordinary construction of governor, a large amount of resistance is opposed by their inertia when they are required to act by rising or falling, on the occurrence of a change in the velocity of revolution. A serious pressure is also caused on the joints of the radius rods when the inertia of heavy balls of $\mathbf{1}$ ewt. each has to be overcome in order to 
accelerate their motion; and the friction caused by this pressure on the joints prevents the change of position of the balls, until a sufficient increase of velocity has occurred to accumulate force enough for overcoming this resistance. The engine is thus allowed to vary considerably in speed; and the balls of the governor are then liable to fly out too far, causing too great an action for properly regulating the speed of the engine. In the present governor the revolving balls being of very small weight offer little resistance by their inertia to any change, and they rise or fall instantly when any change takes place in the speed of revolution of the engine. Their centrifugal foree is made up to that of the ordinary heavy balls by their increased velocity of revolution, the centrifugal force increasing as the square of the velocity; and they are driven at a speed of from 320 to 400 revolutions per minute.

This governor is liable to the same objection in principle as the ordinary Watt centrifugal governor, namely that it can only regulate the engine for a variation of load by maintaining a corresponding change of velocity in the engine; but in this governor the action is so much more sensitive and extended than in the ordinary governor that this objection is practically got rid of. It is found to regulate the speed of the engine with certainty within the range of 2 per cent. variation of speed, with the greatest extent of variation that can occur in the load; and a variation in speed of 5 per cent. would carry the governor through its entire range of action, and shut off the steam from the engine. In practice the steam stop-valve is always set wide open, and the engine runs under all circumstances with complete steadiness and uniformity of motion, without requiring any attention; and the most sudden and extreme changes of load do not affect its motion perceptibly.

Special Construction.-In practically carrying out the high speed of working that has been adopted in this engine, special attention has been required to ensure the rigidity of both the stationary and the moving parts, to balance the forces of the moving parts, and to obtain a large extent of hardened rubbing surface, with perfect truth of form in the wearing parts. When due attention is paid to these points it 
is found that there is no practical difficulty attending the employment of the high speed; and the objections ordinarily felt to it arise really from imperfections of construction in these respects.

For the purpose of obtaining the required rigidity in this engine, the base-plate is made a hollow casting of great stiffness and unusual depth, and the centre line of strain of the engine is brought down very near to its surface. The cylinder is bolted to the end of the base-plate, and is held all round the circumference of its inner end; but it is left free from the base-plate throughout its entire length. The object of this arrangement is to leave the cylinder free to expand and contract, without the tendency to distortion which arises when the cylinder is fixed down throughout one side to a cold base-plate, whilst kept heated along the other side by the steamchest. The cylinder thus preserves its parallelism when at work, so that a deep and well fitted piston can be used; the piston employed is a plain hollow block, turned a close fit to the cylinder, and fitted with two Ramsbottom rings. The result is that, instead of the injurious wear often experienced in horizontal cylinders working at the lower speeds, the cylinders of these engines are found to be always in a polished and greasy condition, and their wear is inappreciable.

In all the working parts of the engine the bearings are made both unusually long and large in diameter, and by this means the pressure per square inch on the bearing surfaces is diminished, so that a thicker film of oil is maintained between them, reducing the coefficient of friction. The smoothness of running is thus increased also, by avoiding the injurious effects that arise from the bearings not having sufficient rigidity to resist flexure or torsion. In all the bearings special care is taken to obtain perfect truth of form, which is a point of great practical importance; and if properly formed and hardened these bearings should not be subjected to wear at all. The difference in working is remarkable between a true cylindrical form and such an approximation to it as can be produced by turning in a good lathe. When a truly cylindrical journal or pin has been produced by the operation of grinding with a traversing wheel, in dead centres which have themselves been ground to true cones, such a cylinder, if it has 
sufficient surface and rigidity and is fitted in proper bearings, floats in an oil bath, being separatel from the bearings at every point by a film of oil of exactly uniform thickness; this film cannot anywhere be broken, and having scarcely any disposition to work out will last without renewal for a great length of time; and the coefficient of friction under pressure is thereby greatly diminished. This truth of form is really easy of attainment, and if its value were fully appreciated it would certainly be obtained in general practice.

Mr. Porter said he had hoped to be able to give the results of a series of comparative trials with one of the Allen engines, as compared with an ordinary engine of good construction and of the same power, working unde' $r$ the same circumstances. It had unfortunately been impracticable however to get these trials carried out yet; but it was intended to proceed with a very thorough set of experiments for this purpose.

Mr. E. A. Cowper enquired what were the particulars of the ordinary engine, with which it was proposed to compare the Allen engine in the experiments; whether it was provided with a separate expansion slide-valve, and what was the degree of expansion employed, and also what was the construction of the air-pump, and whether the cylinder had a steam jacket.

Mr. Porter replied that the other engine was an ordinary horizontal high-pressure engine with an 18 inch cylinder, which had previously driven the shop for three years at the Whitworth Co.'s works, and it had been fitted with a condenser and air-pump exactly the same as those of the Allen engine; so that the comparison would not be complicated by the degree of condensation or the power required to work the air-pump. The cylinder of the engine had not a steam jacket. The steam was cut off at half stroke, and the slidevalve was an ordinary slide cutting off by the lap, without a separate cut-off slide on the back : the object being to make a comparison 
between the Allen valve motion with variable expansion regulated by the governor, and the ordinary slide-valve worked by a single eccentric, with the governor acting upon the throttle-valve in the steam pipe.

Mr. E. A. CowPER thought that for a satisfactory comparison the ordinary engine should be furnished with a separate expansion valve, as was now the common practice in good engines, and also the cylinder should be provided with a steam jacket.

Mr. C. W. Siemens remarked that, as the value of any comparison between the Allen engine and an ordinary engine would depend altogether upon the particular condition of the latter, it would be mach better to have the results of the Allen engine stated independently, giving the consumption of steam per horse power of the work done; this would afford the means of comparing the performance generally with that of any other engines. He was much struck with the very complete manner in which the various mechanical details in the construction of the engine had been worked out; and by the plan of carefully adjusting the weight of the reciprocating parts, so that their inertia at the commencement of the stroke should just counterbalance the highest steam pressure, there was no doubt that a very perfect result would be obtained, provided the speed was always maintained at the full rate upon which the calculation of the inertia was based. The main question he thought was whether the original fine adjustment of the working faces, particularly of the cylinder and slide-valves, could be practically maintained in continued work at the high speed at which the engine had to run; for although the wear of these surfaces might be reduced to a very great extent even at such a high speed, by great care in working and by the use of steam perfectly free from priming, yet it was hardly to be expected that these conditions conld be constantly ensured in actual working for a length of time. It was therefore desirable to ascertain by actual observation what would be the result of continued working with a full load upon the engine. In the engine exhibited last year in Paris it appeared the bearing surfaces had not been altogether suited for the heavy load for which the power of the engine was calculated; and the results of the trials 
with that engine had not been so satisfactory as they would have been, if care had been taken to increase the extent of the bearing surfaces in proportion to the load put upon the engine.

The governor described in the paper would he thought certainly be quicker in its action than the ordinary arrangement; and no doubt if an ordinary ball governor were weighted down in a similar manner by a weight or a spring, and had its speed accelerated to the same extent, its quickness of action would be correspondingly increased. The improved governor was in fact a Watt governor revolving at a higher speed; and it was therefore necessarily subject to the defects attending the principle of that governor, namely that at the moment when the engine began to change its speed the governor was without any power to act upon the valve, and it had then to accumulate power itsclf before it could control the engine. He should have felt some hesitation in going to such a high speed with the revolving ball governor, for fear of rapid wear and tear; but this was a point which would be decided by the results of actual working:

Mr. F. W. WebB concurred in the opinion that the most useful criterion of the economy of the Allen engine would be the actual consumption of fuel per horse power; and from his own experience of high speeds he agreed in thinking there would be a difficulty in maintaining the wearing surfaces of the slide-valves and cylinder, so as to keep them steam-tight in continued working.

Mr. E. Reyrougs said ho had seen the Allen engine at work at Manchester on several occasions, and he had also seen the ordinary engine that had been mentioned, with which it was proposed to make the comparative trial; and from the results said to be obtained with it he thought the latter might be taken as a very favourable example of an ordinary condensing engine. Simplicity of construction was a very important consideration in an engine for common use; and if an ordinary horizontal condensing engine, with a single slide-valve cutting off at half stroke, without a separate expansion valve, and without superheating the steam, could be made to work with 3 lbs. of coal per horse power per hour, which he understood was the case at the Whitworth Co.'s works, a vey high result was attained. 
The effect of the inertia of the reciprocating parts was a matter of great practical importance in any high-speed engine; and although the general principle had long been admitted, it did not appear to have been fully understood previously, and at any rate had not been fully acted upon in practice hitherto. In the present instance the effect of that inertia appeared to have been arrived at by a tentative process, by calculating the amounts of acceleration and retardation through each degree of revolution of the crank, and then the forces necessary to produce them. The simple way however to calculate the actual effect produced upon the crank pin by the inertia of the reciprocating parts in a horizontal engine seemed to him to be by supposing the whole weight of the horizontal reciprocating parts to be concentrated at the crank pin; and the centrifugal force due to the revolution of this weight in the circle of the crank being then resolved at any point of the circle into the vertical and horizontal directions, he considered that the horizontal component would represent the actual effect produced upon the crank pin at that point by the inertia attending the horizontal reciprocation of the same weight if accelerated and retarded in precisely the same degree and manner, though having horizontal motion only. In this mode of calculation the line representing the force necessary to produce the acceleration of the reciprocating parts by the crank in the first half of the stroke and their retardation in the latter half, during equal spaces passed through by the piston, would be a straight line corresponding with that described in the paper and shown in the diagram (Fig. 17), for a connectingrod of infinite length; the maximum effect upon the crank pin being at the commencement and end of the stroke, at the moment when the crank was passing the centre, while the effect of the inertia became nothing at the middle point of the stroke. The effect of taking into account the length of the connecting-rod would be obtained by considering in an exactly similar manner the centrifugal force due to the angular motion of the rod in its arc of vibration, the horizontal component of which at every point of the stroke would have to be added in the outer half of the stroke to that previously ascertained with an infinite length of rod, and dedueted in the inner 
half. At the two extremities of the stroke therefore, the lineal velocity of the crank pin in its circle of revolution and of the connecting-rod end in its are of vibration being at that moment identical, the amounts of centrifugal force due to the crank and to the connecting-rod would be in the inverse ratio of their lengths; and in the case of a connecting-rod having six times the length of the crank, the centrifugal force due to the connecting-rod would thus cause an addition or deduction of one-sixth upon that due to the crank. The total pressure produced on the crank pin by the inertia of the reciprocating parts would accordingly be seven-sixths at the outer extremity of the stroke and five-sixths at the inner extremity, in comparison with the equal pressure at both ends due to a connecting-rod of infinite length ; thus giving a difference of $16 \frac{2}{3} \mathrm{per}$ cent. above and below the mean of the two, which was the same result as that obtained in the paper by a different method of calculation. On account of this difference it was clear that no single counterbalance weight, such as that added upon the driving wheel of a locomotive engine, could accomplish a true balance throughout the whole stroke; the utmost that could be done by such means was to balance the mean inertia, leaving the variation between the two extremities of the stroke without compensation. Although with the proportions and counterweights adopted in most engines at the present time this variation was not so considerable as to be of much importance, he thought it was still worth while to draw attention to the fact, in order to show the futility of attempting an absolutely true balance. This attempt had been made in the "duplex" locomotive engine shown at the International Exhibition in 1862 (see Proceedings Inst. M. E. 1863, page 97), one piston at one end of the stroke being intended to balance the other piston at the opposite end. But even in that case, though the difference in pressure of the wheels upon the rails due to the rertical action of a revolving connterweight in the wheel was avoided, yet the inequality in the inertia in the two halves of the stroke was still left untouched; as was also the vertical pressure of the crossheads against the slide-bars, tending to lift the two sides of the engine alternately : and no nearer approximation to a true balance was really effected than in ordinaryengines having counterweights 
attached to the wheels. In locomotives, in consequence of the variable speed as well as this difficulty in balancing, he thought the best mode of attaining steadiness in running was to keep the pistons and other reciprocating parts as light as possible; but in stationary engines intended to run constantly at a given uniform speed, it was most advantageous, as shown in the paper, that these parts should have a considerable amount of weight, so as to give the amount of inertia calculated to correspond with the standard speed of working.

The importance of bestowing sufficient attention upon the details of an engine appeared to him to be well illustrated by the working of the Allen engine at Messrs. Pooley's cotton mill in Manchester, which he had recently seen at work doing a heavy duty perfectly well, and with complete freedom from any signs of heating or undue strain. In general when it had been attempted to run a horizontal engine continuously at a high speed under a heavy load, the result had been that the bearings became very hot, unless a great deal of play were allowed; and on this account all previous attempts that he was acquainted with of running such engines at a speed anything like 600 feet per minute of the piston had been attended with much trouble and annoyance. At that speed however, and even higher speeds, the Allen engine worked quite comfortably, owing probably in a great measure to the special care taken to provide ample bearing surfaces and to get them up truly cylindrical. Another part of the engine which had been well worked out in the details was the air-pump. There was perhaps nothing strictly new in the plan of air-pump adopted for the Allen engine, as the importance of getting a large extent of surface for the water with small extent of motion had been previously advocated, instead of having the area of the water surface only the same as the area of the piston, as in ordinary air-pumps; nevertheless high-speed air-pumps had always been a difficulty, as shown by the violent oscillations of the vacuum gauge even under the best arrangements previously tried. In the ordinary engine however which he had seen at the Whitworth Co.'s works, fitted with the improved air-pump described in the paper, the vacuum gange remained remarkably steady with the cock wide open, even 
when the engine was running at the high speed of 94 revolutions per minute; at that speed there was only a slight pulsation in the gauge, instead of the sudden oscillations which would be exhibited with an ordinary air-pump driven at a considerably lower speed. This result showed that attention to the details was of the highest importance for the efficient working of an engine; and the same remark applied to the plummer blocks for carrying the main driving shaft, which he thought were well arranged for getting over the difficulty often experienced in horizontal engines of avoiding horizontal play without inducing heating of the bearings.

In regard to the improved construction of governor described in the paper, he thought that although an ordinary Watt governor if loaded in the same proportion might theoretically be considered to be equivalent in action, jet in that case the friction on the collars would necessarily be so great as to require a very large power to drive it; whereas by the device of taking so much weight out of the balls themselves, and applying it instead in the shape of a central weight lifted by the toggle-joint, the inertia of the balls was so far reduced that they offered much less resistance to any rise or fall corresponding to a change of speed. A less amount of power would therefore produce a given rise of the light balls in the improved governor, though lifting a weight equal to that of the heavy balls in an ordinary governor. On this account, and considering also the other improvements in the detail of the governor, he could believe that the extreme variation of speed in an engine controlled by this governor would not exceed 2 per cent. Having himself used many of these governors, he had found their increased sensitiveness rendered the speed of the engines remarkably steady; and as a cheap and simple construction of governor, sufficient for all ordinary circumstances, he considered the improved arrangement was a very valuable contrivance.

The Chatrman remarked that the consumption which had been mentioned of only 3 lbs. of coal per horse power per hour, by an engine expanding only twice, was a very remarkable result; he had never heard of such a performance before, and would be glad to know whether the statement had been verified. 
Mr. E. Rerrolns said that was stated to be the consumption of the ordinary engine at the Whitworth Co.'s works. It did not follow however that, because the slide-valve cut off at half stroke, the expansion was only twofold; for though the steam was throttled in the steam pipe, it had ample time to accumulate in the steam. chest during the latter half of each stroke, after it was cut off from the cylinder; so that the full pressure of steam came upon the piston at the commencement of each stroke, and was very rapidly reduced as the stroke progressed by wire-drawing through the throttle-valve, in consequence of which the resulting indicator diagrams were not very different from those produced by true expansion with a considerably earlier cut-off.

Mr. PORTER explained that the consumption of $3 \mathrm{lbs}$. of coal per indicated horse power per hour in the ordinary engine at the Whitworth Co.'s works was exclusive of raising steam at starting in the morning; the temperature of the feed-water supplied to the boiler during working was below $100^{\circ} \mathrm{Fahr}$. This result had been obtained in a week's careful experiment, and he believed its accuracy was unquestionable. The fuel used was the ordinary Lancashire coal, which was so much inferior in heating power to the Welsh, that the consumption of $3 \mathrm{lbs}$. per indicated horse power would be equivalent to only about $2 \frac{1}{2}$ lbs. of good Welsh coal.

Mr. F. A. CowPen remarked that he could not approve of the Allen engine, but on the present occasion he did not wish to go into the questions of the high speed of piston or details of the engine, and would only refer to the subject which had been alluded to of the plus and minus pressure exerted on the crank pin by the momentum of the reciprocating parts. When the velocity of the piston was changing from an accelerating to a diminishing velocity in the middle of the stroke, there was no plus or minus pressure on the crank pin; but when the crank had passed the end of the stroke and the velocity of the piston was increasing, there was a minus pressure, that is, a portion of the pressure of the steam on the piston was absorbed in getting up its velocity and was not at that time developed on the crank pin; and then again in the latter portion of the stroke there was a plus pressure or extra pressure on the 
crank pin over and above the pressure of the steam, from the fact of the crank pin then retarding and changing the velocity of the piston. If the ordinates of this plus and minus pressure were set out to form a diagram, they would give a curve dying away to nothing at about half stroke.

He wished to enquire whether it was intended that, on acconnt of the very high speed of piston in the Allen engine, a small-cylinder engine on that construction should be considered as one of large power.

Mr. L. Otrior said he had seen the Allen engine at work at the Paris Exhibition, and also the very excellent sectional working model that was shown there, which gave a very clear idea of the valve motion worked from the single eccentric. By the very simple and ingenious arrangement of the bell-crank levers working the two slides, the object was attained of a sharp cut-off, without contracting the port openings by the gradual closing of the valve, as was the case with an ordinary slide-valve driven by an eccentric; and a very free opening was afforded for the admission of the steam, which entered at four distinct places simultaneously; while the entire valve motion was worked by a single eccentric always in gear. In respect to the governor described in the paper, there could be no doubt that the chronometric governor of Mr. Siemens was quicker in action and the nearest approach to absolute perfection; that governor was however rather more complicated in construction, and the ballgovernor now described was certainly very simple and sufficiently perfect for most ordinary engines ; for although in principle it was a Watt governor and involved therefore the same fundamental defects, these were reduced to a very small actual amount, by the improved canstruction of the parts and the use of much lighter balls revolving at a much higher speed. By the working of the Allen engine the very high speed of piston mentioned in the paper had now been shown to be practicable, without involving the objections usually experienced. hitherto in any attempts to increase the speed of piston considerably; and he thought the successful results now attained were satisfactorily accounted for by the explanation given in the paper, the weight of the reciprocating parts being angmented to such 
an extent that their inertia entirely absorbed all the initial surplus pressure of the steam on the piston at the beginning of each stroke, thus relieving the crank pin from the usual great strain on the centres, which would otherwise be most dangerous with such quick-working engines. This principle having been so carefully carried out in the proportions adopted in the Allen engine rendered it a race-horse in speed as compared with ordinary engines; and for such an engine the lighter and more sensitive governor of Mr. Porter was excellently adapted. They were also indebted to him for the introduction into this country of the Richards indicator, the lighter and more sensitive construction of which rendered it the only instrument capable of giving a correct diagram from an engine running at a very high speed. The remarkable attention paid to the details in all the parts of the Allen engine might be imitated with great advantage he thought in all other descriptions of machinery, the ordinary turned bearings being only approximately cylindrical, and in reality more or less polygonal; but it was only in conjunction with ample extent of surface and perfectly round bearings that the high speeds of working described in the paper had been so successfully realised.

Mr. H. Woons asked if it was intended in the construction of the Allen engine in the future to discontinue the use of the steam jacket.

Mr. J. FERnIE said he had seen the Allen engine when first shown at the 1862 Exhibition, and also the improved one at the Paris Exhibition and the one working in Manchester, and he had been much pleased with their performance; they were very steady at the great speed at which they ran, and seemed to do the work with remarkable ease. One reason of the smoothness of running was that there was no feed-pump attached to the engine, and the rattling action attendant upon a pump was thereby avoided. The speed of piston, although much higher than was usual in stationary engines, was not more than was general in locomotives; for a locomotive running at 40 to 50 miles an hour had a piston speed of about 600 feet per minute. With the adoption however of this high speed in stationary engines, he suggested that it would be very 
desirable at the same time to increase the pressure of steam up to 100 or 150 lbs. per square inch, in accordance with the present practice in locomotives; if this were done, and the principles already carried out in the Allen engine were as carefully adhered to with the higher pressure, he thought the results obtained would be still more advantageous. He enquired what was the amount of lead given to the slide-valve, in working with the single eccentric; and also what was the practical advantage afforded by the engine, in respect to the proportion between the power ubtained and the space occupied.

Mr. A. Coventry, having had several opportunities of seeing the Allen engine at work in Manchester and in London, considered the most distinctive feature about it was that it took up all the best points of other engines, and combined them in one engine. A quick cut-off was obtained, with a variable expansion regulated by the governor according to the load on the engine; at the same time the exhaust was invariable at all grades of expansion, and very free. It was also deserving of remark that for an engine combining all these advantages it contained the smallest possible number of parts.

Mr. PorTer remarked that the piston speed of 800 feet per minute in the Allen engine at the Whitworth Co.'s works was considered exceptional; and the standard speed in calculating the proportions of the engine had been taken at 600 feet per minute. At the same time if a higher speed, even up to $\mathbf{1 0 0 0}$ feet per minute, were desired for any special purpose, he shonld not hesitate to run the engine at that rate, with suitable modifications in the proportions of the parts.

In reference to the heating of one of the bearings in the engine at the Paris Exhibition, as mentioned in the paper, that was not owing to want of sufficient extent of surface, but was altogether an accident arising from an error in the bearing, which caused it to be imperfectly lubricated. The dimensions of the bearings were very large; and the engine at the Whitworth Co.'s works, with the same dimensions, had been working very satisfactorily under a load twice as heary as that put ujon the engine in Paris. With the ample extent of bearing surface that was provided, it had been 
found that the engine was perfectly safe from heating, so long as uniform lubrication was ensured. On the engine at Manchester all risk of imperfect lubrication was prevented by the employment of a continuous mechanical lubricator, consisting of a brass cup fitted with a cock having a solid plug, in one side of which was drilled a hole large enough to hold a couple of drops of oil, so that at each revolution of the plug that quantity of oil was supplied to the crank pin; the plug was caused to revolve by means of a worm and wheel, actuated by a small cam let into the circumference of the crank-pin collar. In lubricating with the ordinary cotton wick as a syphon, it occasionally happened that the lubrication failed for a length of time without the defect being observed; but the mechanical lubricator had been found to remove that difficulty altogether.

The lead of the valves was necessarily considerable in running at a high speed, in order to afford a sufficient area of inlet for completely filling the port and clearance space at the commencement of each stroke; and in the original setting of the valves the lead was adjusted with reference to the indicator diagram, until a figure was obtained with a sufficiently square corner at the beginning of the stroke. The amount of lead thus arrived at was such that the steam could not be entirely shut off by the governor, when the work was thrown off the engine; but when the whole load was thrown off excepting only the main line of shafting driven by the engine, the admission of steam was so far reduced by the governor that no acceleration of speed took place in the engine.

For taking up the wear of the slide-valves the requisite means of accurate adjustment was provided in the manner described in the paper, by the insertion of packing strips underneath the supports of the cover-plate on the back of the valves; so that the rubbing faces could be let together at any time to the exact extent required, by simply reducing the thickness of these packing strips. The form of piston to be used was quite immaterial; the piston which he had employed was packed with two Ramsbottom rings, as shown in the drawing, and had been found quite satisfactory. 
The governor controlling the Allen engine was absolutely the same in principle as the original Watt governor; but the object had been to obtain a nearer approach in practice to the degree of perfection of which that principle was capable. This was effected by reducing as far as possible the severe friction upon the joints, which exerted so great a retarding force npon the action of the ordinary governor; and a further step was the substitution of the central heavy weight, instead of putting the whole amount of weight into the revolving balls.

It was the consideration of the inertia of the reciprocating parts which he thought constituted the most important point in the construction of the Allen engine; and in the case of the engine at the Whitworth Co.'s works this ermsideration had justified the adoption of a mean initial steam pressure as high as 57 lbs. per square inch; but were it not for the compensating effect of the inertia of a heavy piston, it would be impossible for the crank pin to stand anything like such a pressure in an engine of that size. By the exact balancing of the initial pressure of steam and the inertia of the reciprocating parts an increased smoothness in running was obtained, and a gliding motion in passing the centres, instead of having a concussion upon the crank pin at these points.

The air-pump did not contain any entirely new features, but was simply a combination of all the best points in previons air-pumps, and he thought it was a very satisfactory solution of the question of working an air-pump in an engine running at a high speed. The vacuum constantly maintained at the speed of 94 revolutions per minute was $27 \frac{1}{2}$ inches of mercury and often 28 inches; and even at 200 revolutions per minute the average vacuum was 26 inches. No trouble whatever had been experienced with the air-pump in regularly working at those high speeds.

The use of a steam-jacket he had been led to abandon in the case of small engines; for although it was unquestionably advantageous wherever there was abundant communication with the boiler, so as to get the full heat of the briler steam in the jacket and allow the condensed water to drain back freely, yet in small engines there was much difficulty in ensuring these conditions being carried out. It 
frequently happened indeed that, owing to the accumulation of water in the steam-jacket, the temperature surrounding the cylinder was considerably lower than that at which the steam entered the cylinder; and under such circumstances the jacket was of course an injury rather than a benefit. In expanding to a very great extent, such as cutting off the steam at 1-10th of the stroke, it was certainly impossible to work with advantage unless the cylinder was jacketted; but on the other hand he did not think it desirable in practice to cut off the steam so early as 1-10th, and he considered a cut off at 1-6th or 1-5th was the earliest that was advisable. Although expansion had great advantages in economy of fuel, it might be overdone, if the limits assigned by other considerations were not attended to.

The degree of accuracy of work required in an engine running at the high speeds named in the paper was nothing more than the best makers of engines and machinery were now thoroughly accustomed to; what would be considered reasonably good work for other similar purposes would do very well for a high-speed engine; but of course any errors arising from inferior work were sooner detected by the high speed, and their evil effects aggravated. In the case of the Allen engine at the Whitworth Co.'s works, the result of the large extent of the wearing surfaces and the excellence of the work had been that, after running constantly for several months, there was absolutely no wear about it at all : the cylinder and slide-valve faces were perfectly smooth, and the guide bars still showed the original marks of the scraping tool.

The commercial benefits attending the Allen engine were not so important a subject for consideration, he thought, as its mechanical advantages; and a reasonably high speed had a great many advantages for stationary engines besides the saving of space and the economy in first cost. In the present instance, with the high speed now employed, the engine had only a single cylinder instead of two cylinders coupled at right angles, and yet gave the same uniformity of motion; it was also a horizontal engine instead of a beam engine, and a simple engine instead of a compound one.

The pressure of steam employed in the Allen engine at the Whitworth Co.'s works, namely 57 lbs. per square inch, although 
higher than hitherto commonly adopted in condensing stationary engines, was by no means so high as he thought might with advantage be used; and he agreed in considering that the higher the pressure, the better would be the results obtained from the engine. The proportions of the several parts would of course have to be modified for an increase of pressure beyond certain limits; and the engines could be constructed to work at any steam pressure that might be desired. The number of separate parts contained in the engine was on the whole smaller, as had been noticed, than in an ordinary engine of the same power; and the same remark applied even more forcibly to the number of working joints in the engine. The joints in the valve gear, which were frequently a source of trouble in other engines, appeired in this to be absolutely free from wear, and had never given the slightest trouble. The air-pnmp being here worked direct from the piston-rod of the steam cylinder was without any joints at all; whereas in an ordinary engine there were commonly several joints involved in the connection for driving the air-pump.

The Chairman proposed a vote of thanks to Mr. Porter for his paper, which was passed.

The following paper was then read:- 


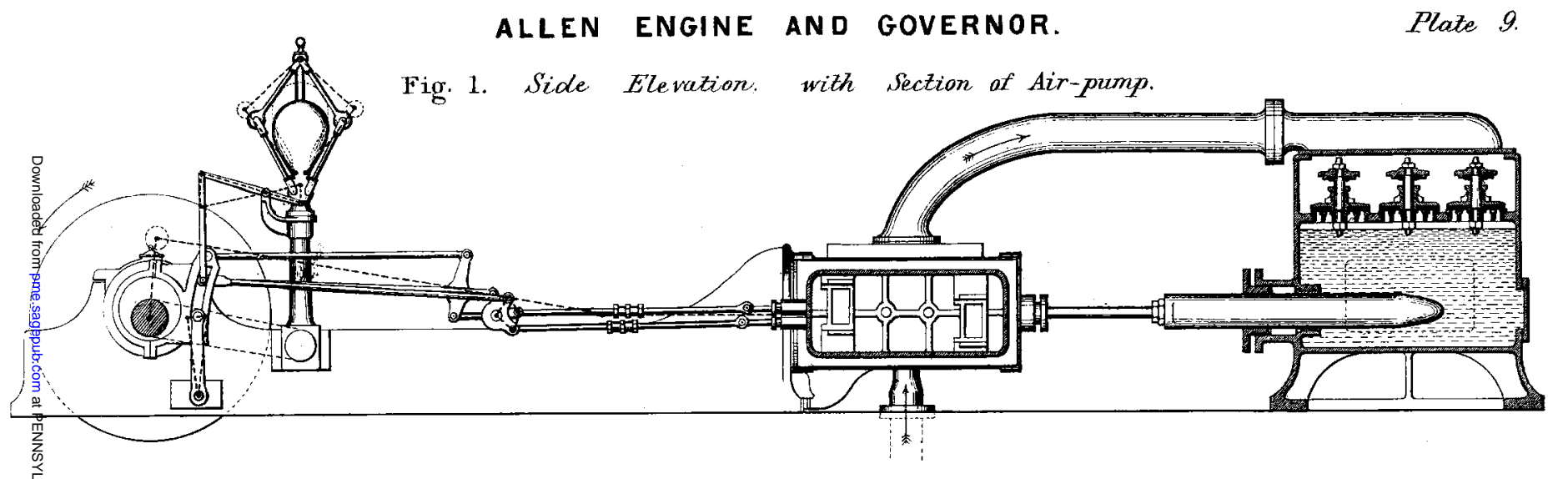

\section{Fig. 2. Plan}
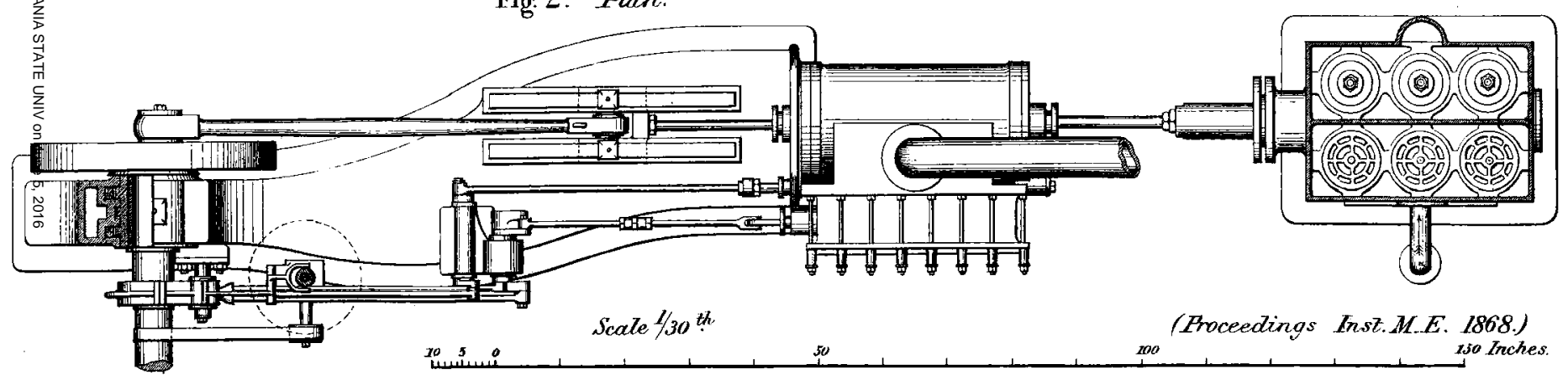

iII

(Proceedings Inst.M.E. 1868) ${ }_{350}$ Inches 


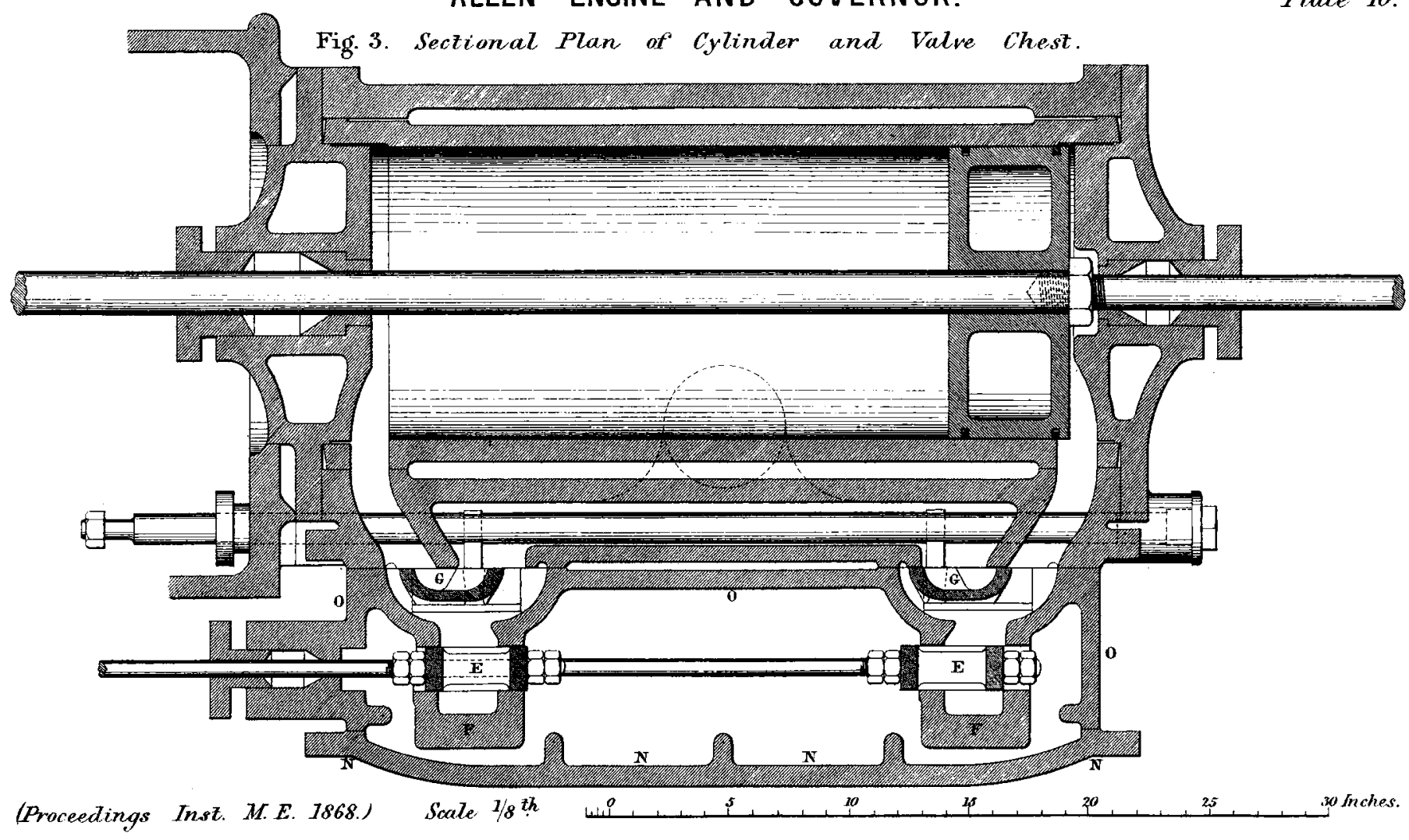


ALLEN ENGINE AND GOVERNOR.

Fig. 4. Transverse Section

Elevations of Port Faces.

Plate 11 .
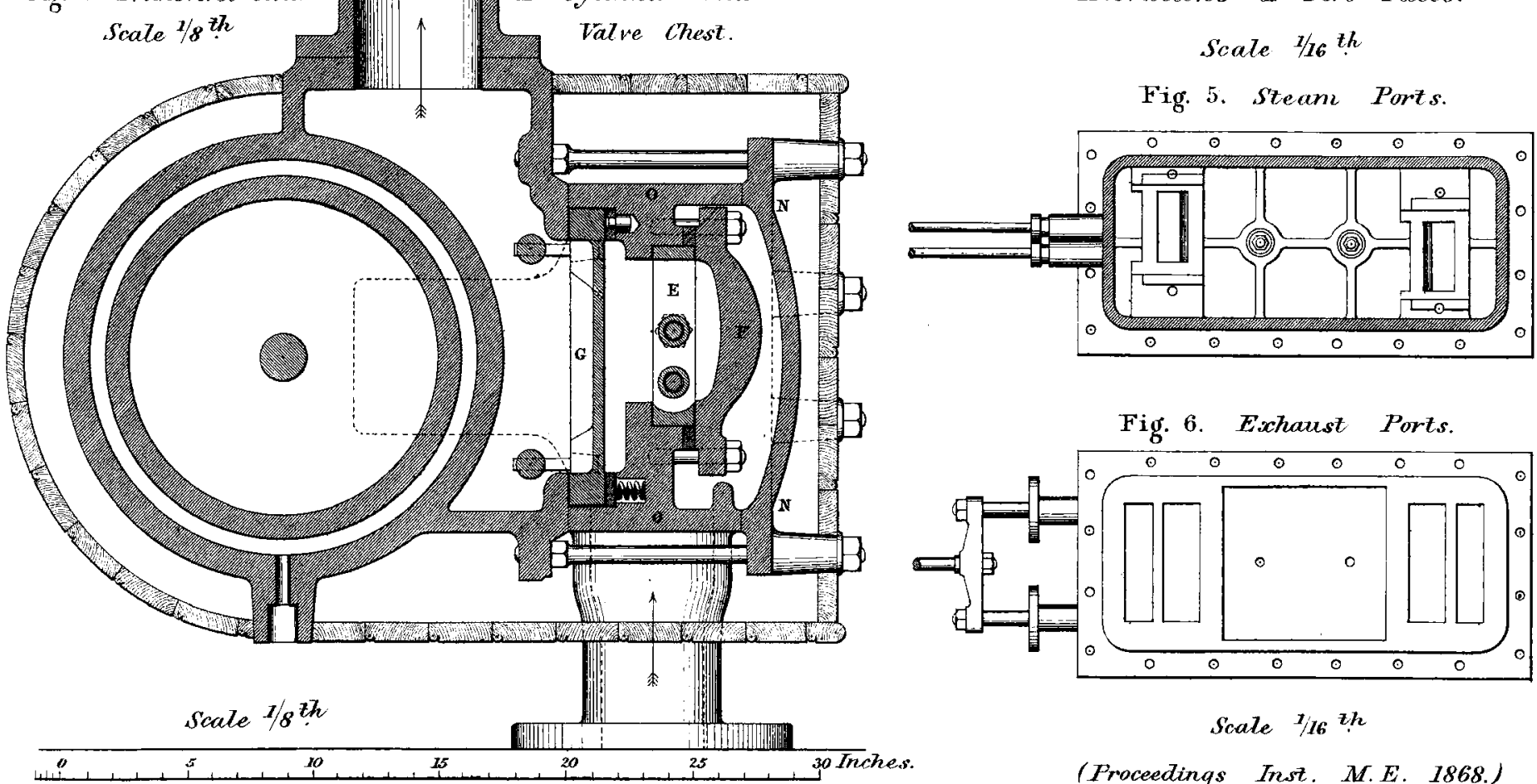

(Proceedings Inst. M.E. 1868.)

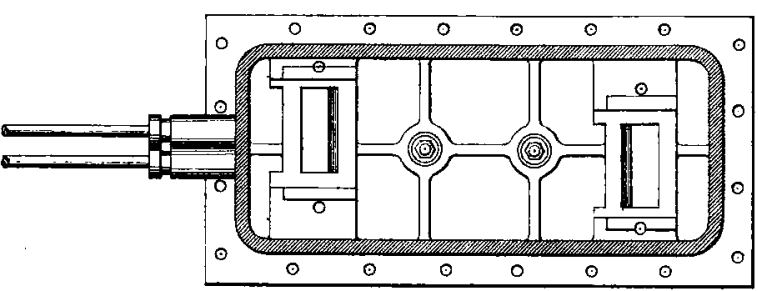


ALLEN ENGINE AND GOVERNOR. Plate 12.

Fig. 7. Thansverse Section of Condenser and Air-pump.

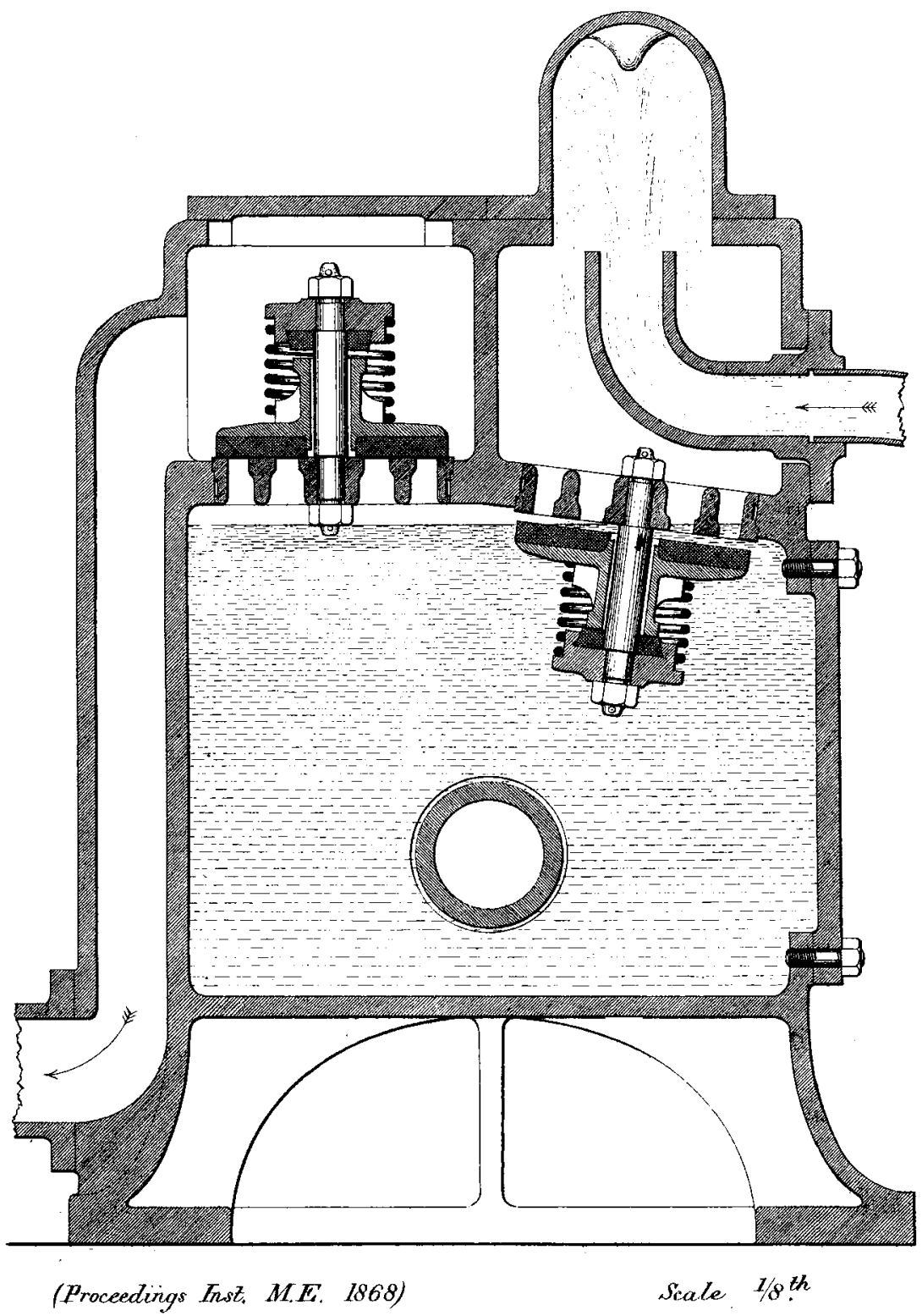


ALLEN ENGINE AND GOVERNOR. Plate 13.

Elevations of Governor.

Fig. 8 .

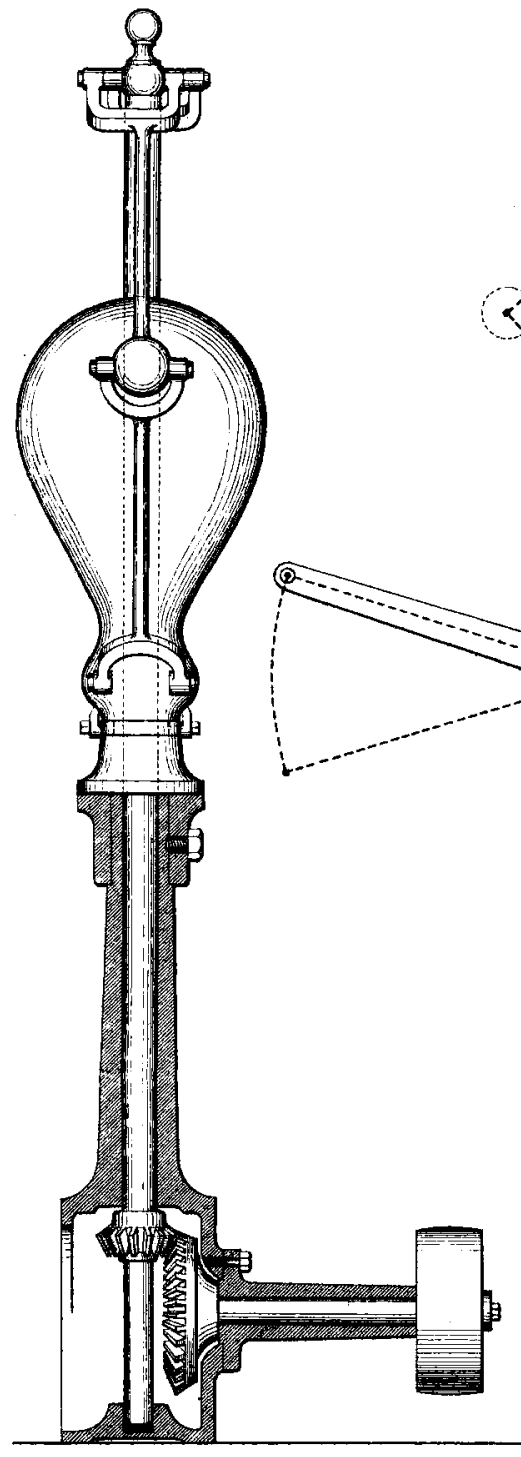

(Praceedings Irost. M.E. 1868)
Fig. 9 .

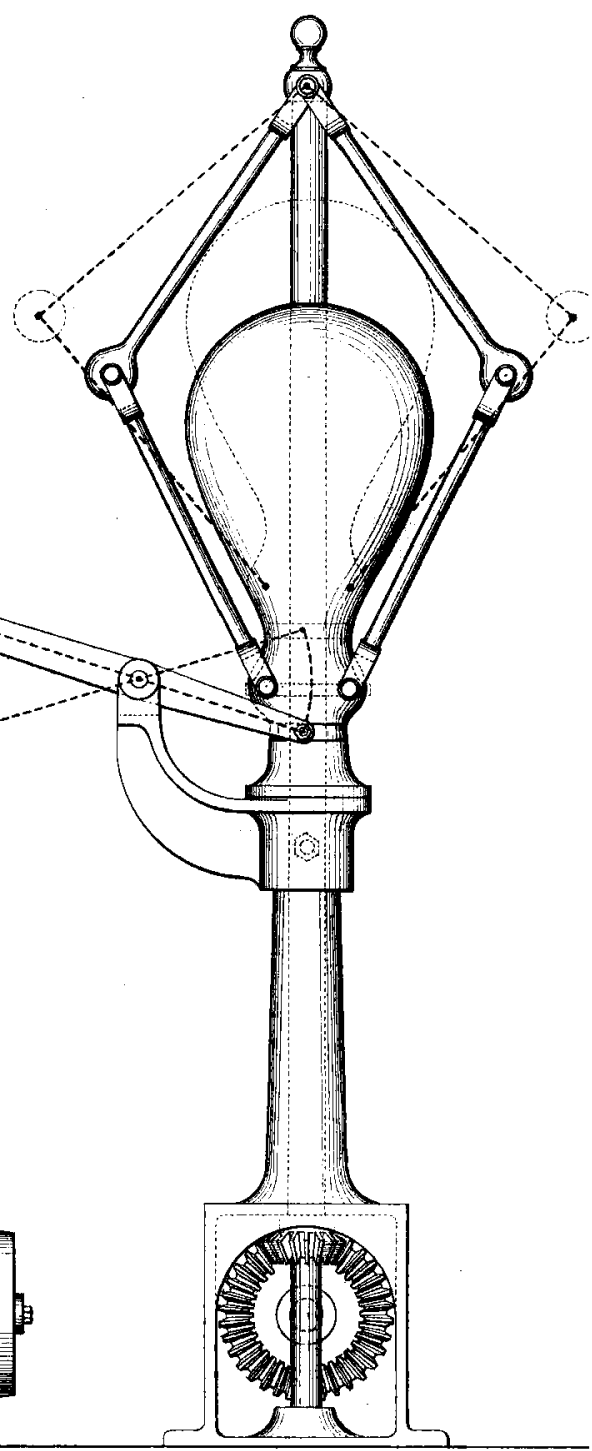




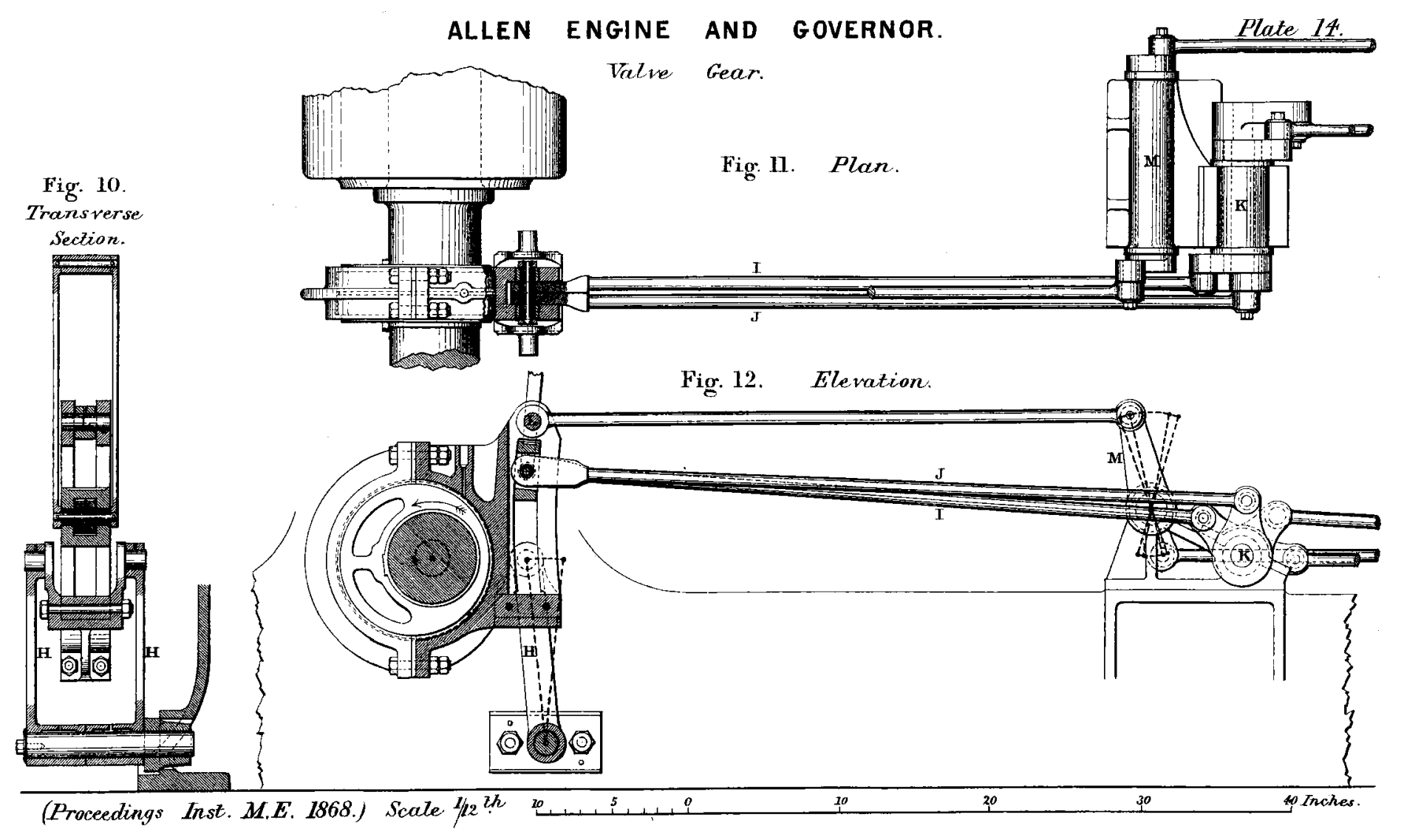




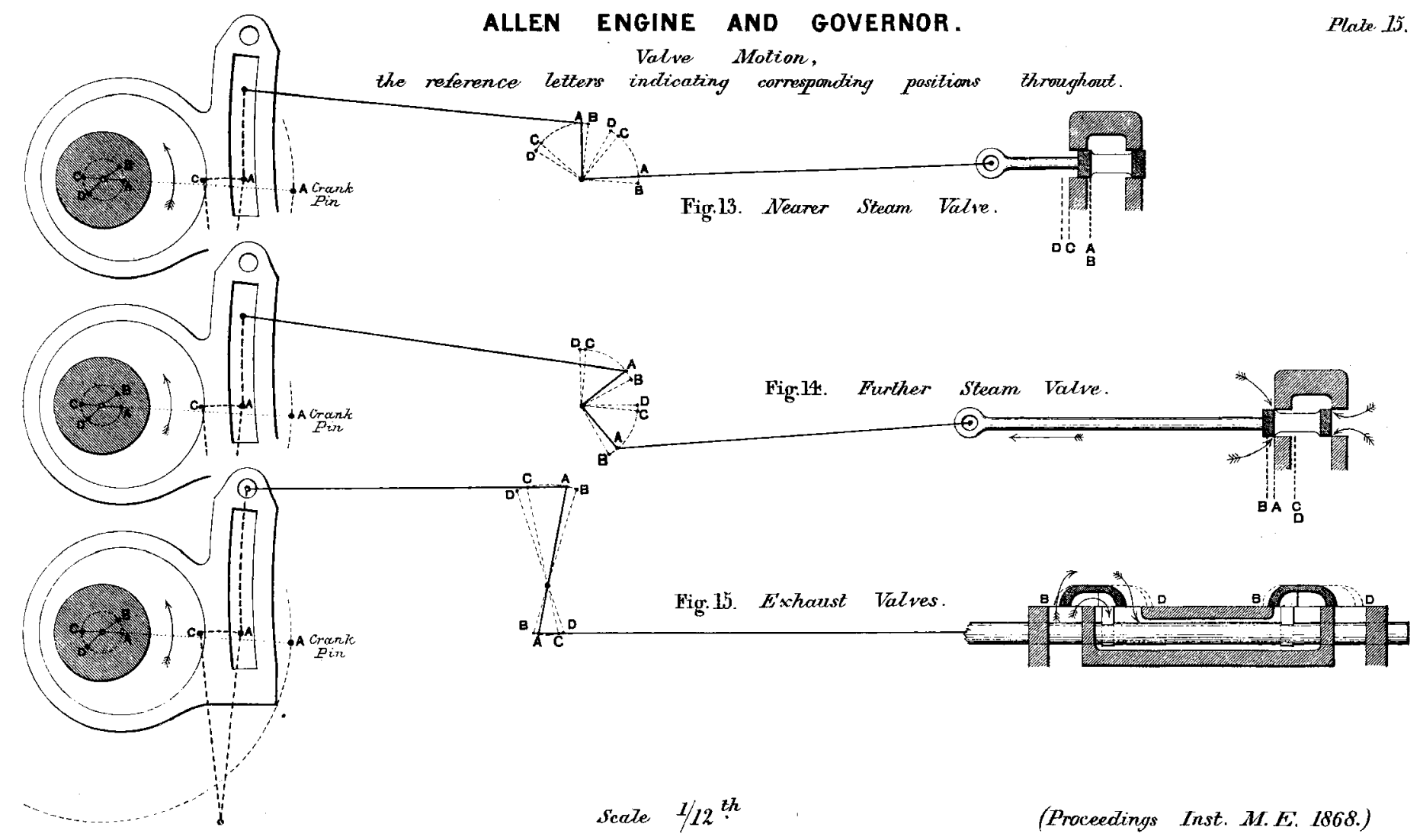


ALLEN ENGINE AND GOVERNOR.

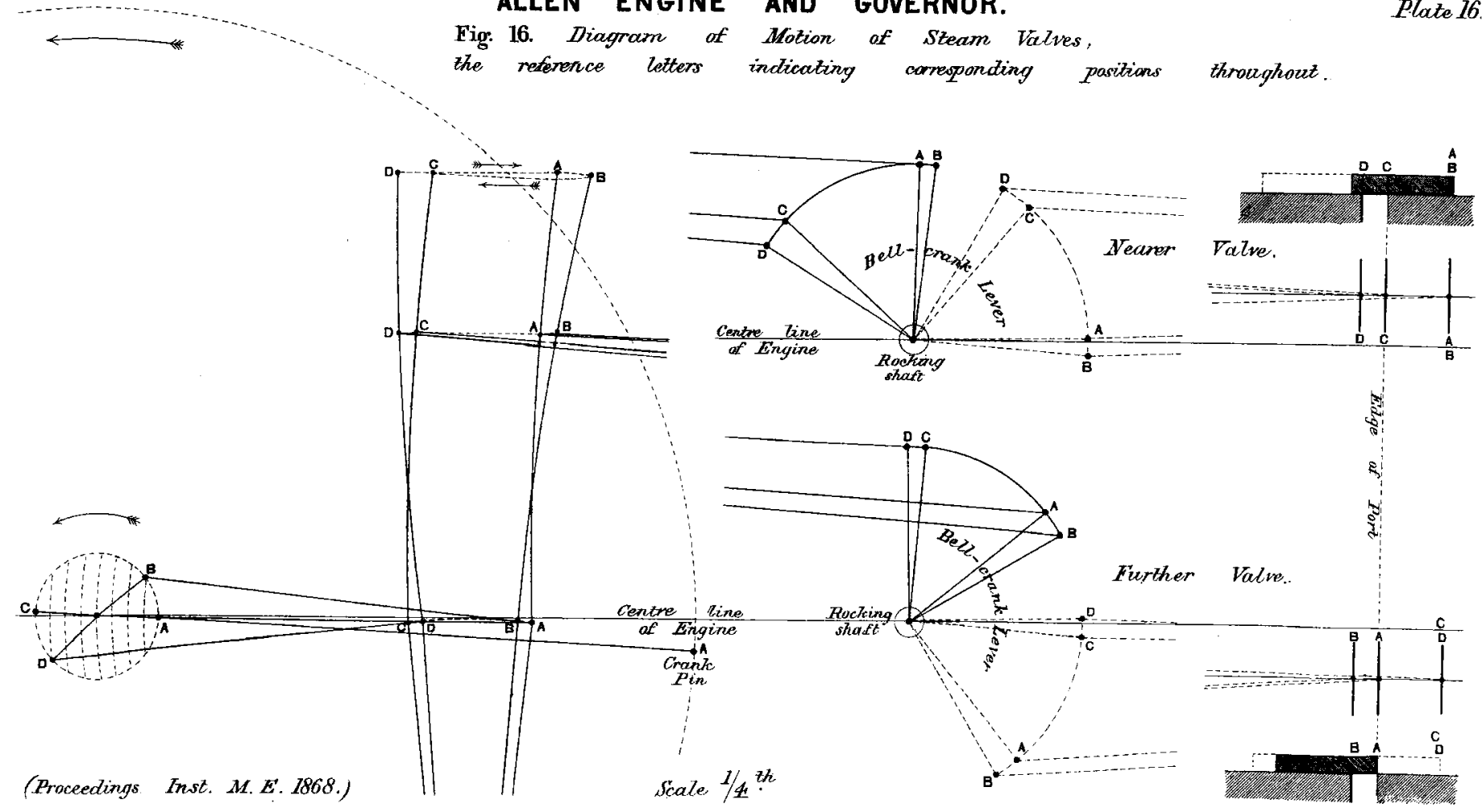




\section{ALLEN ENGINE AND GOVERNOR.}

Fig. 17. Diagram showing Pressure upon Crank Pin,

produced by the inertia of the horizontal reciprocating parts

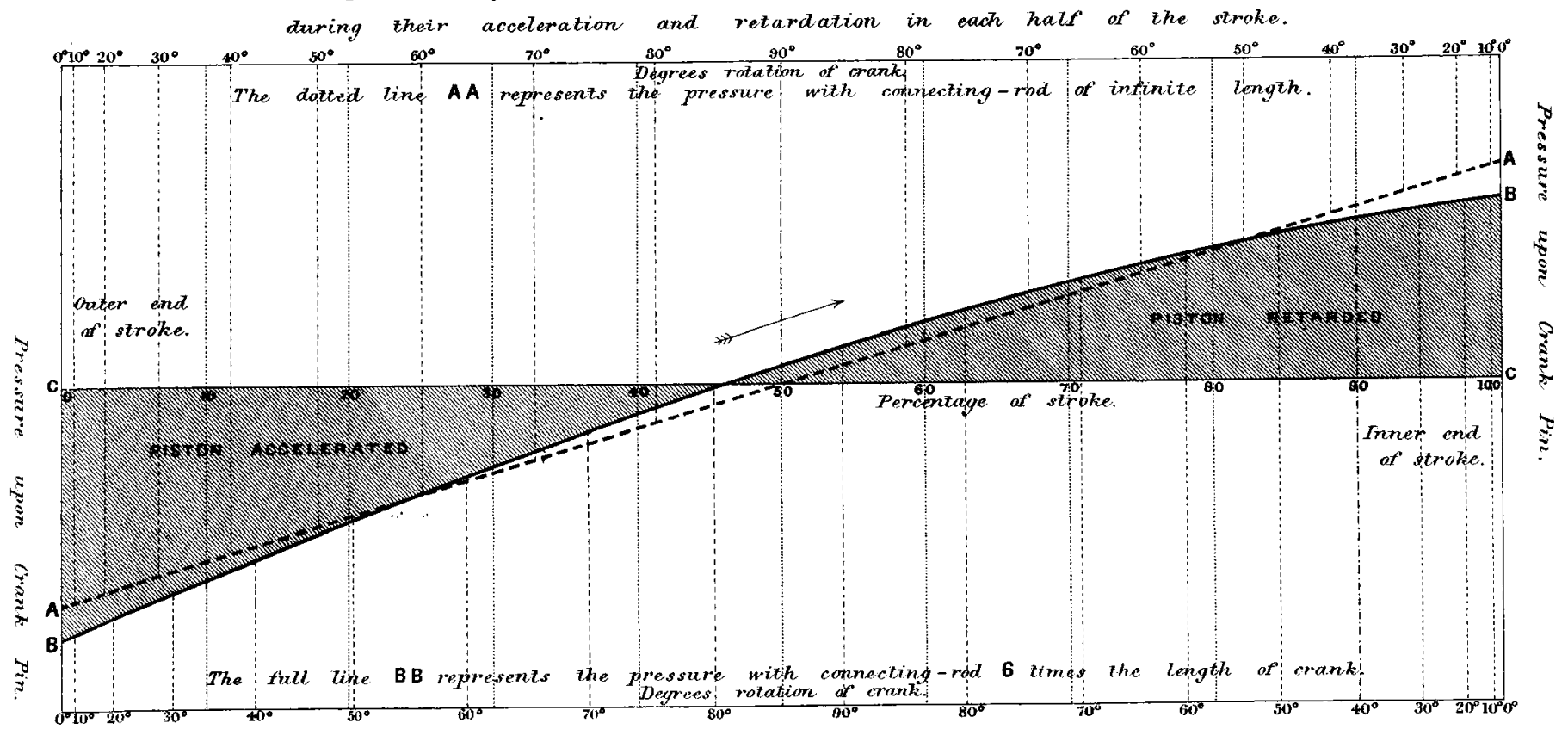

(Praceedings Inst. M.E. 1868.) 
ALLEN ENGINE AND GOVERNOR. Plate 18.

Fig. 18. Indicator Diagrams from Allen Engine.

Cylinder 12 ins. diam., 24 ins. stroke.

200 Revolutions per minuze.

Speed of Piszon 800 feet per minuze

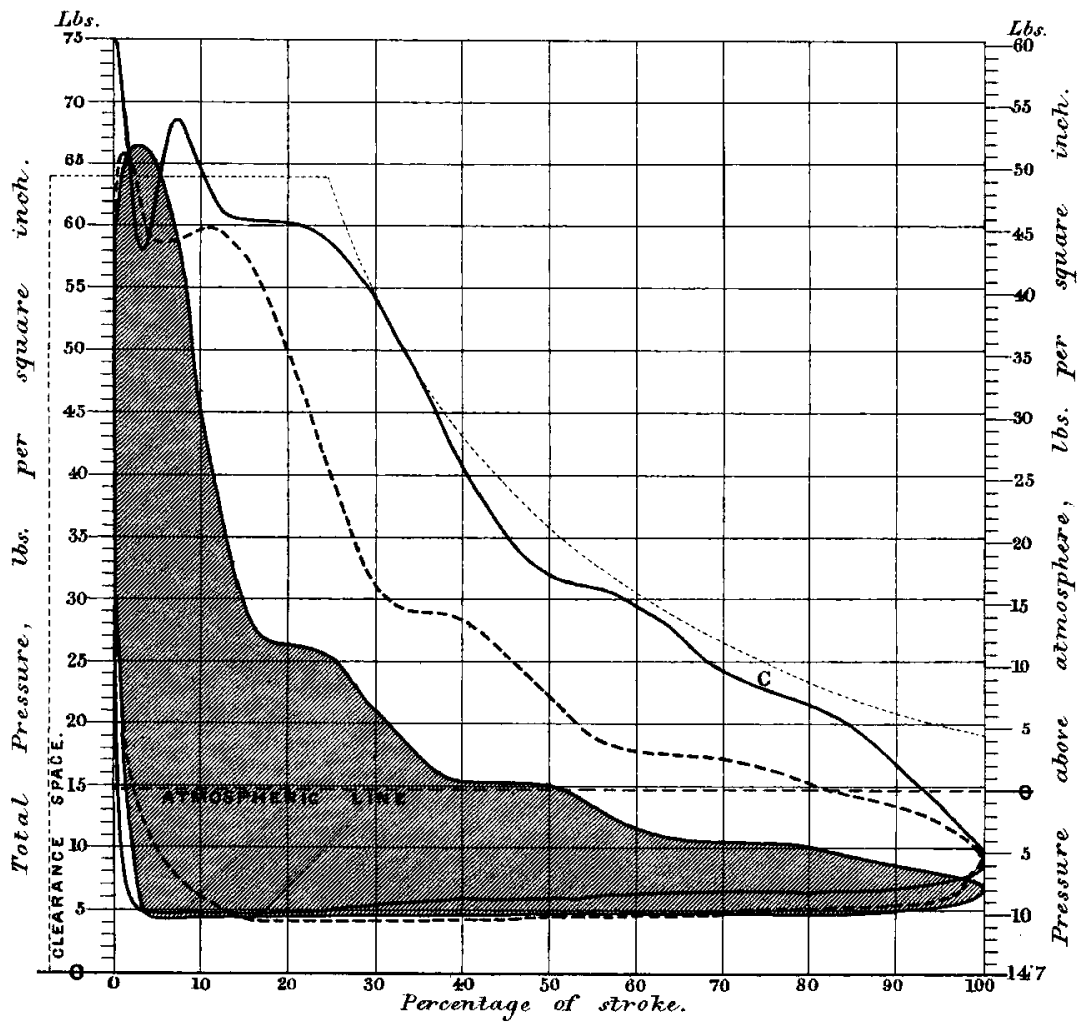

The fine dotted line shows the theoretical true expansion carve of the indicutor diagram $\mathrm{C}$.

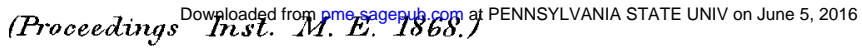




\section{ALlen engine AND governor. Plaze 19.}

Fig. 19. Indicator Diagrams from Allen Engine

Cylinder 18 ins. diam., 30 ires. stroke.

120 Reroluzions per minaze.

Speed of Piston $\mathbf{1} 10$ feet per minute.

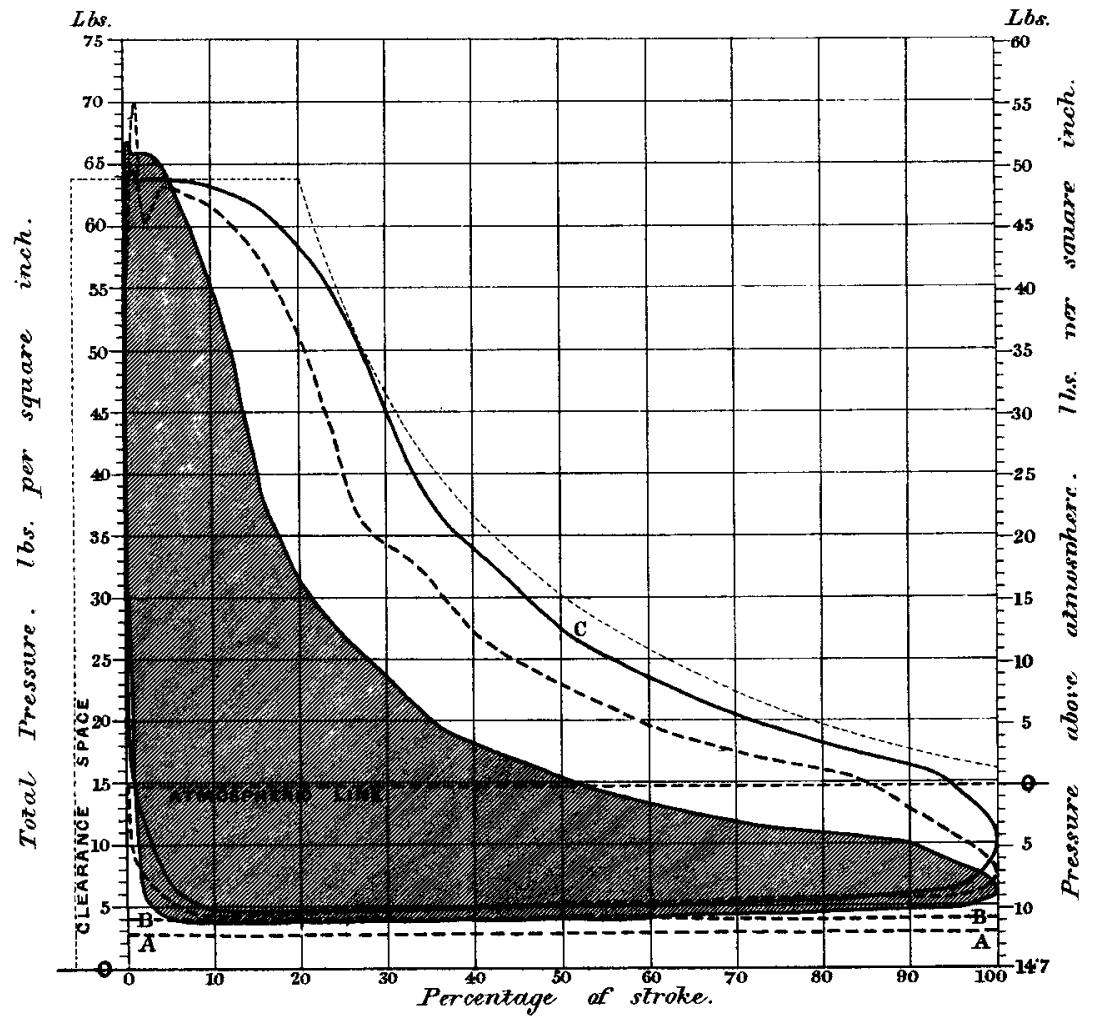

AA rrowam in rondenser \}at the time of zuking the

BB .. $\quad . . \quad$ exhaust chamber $\}$ dotted indicator diagram.

The fine dotzed ture stows the theoretical true expansion curve of the indicutar diayrame $\mathrm{C}$.

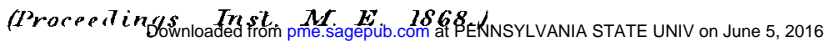




\section{ALLEN ENGINE AND GOVERNOR. Plaze 20.}

Fig. 20. Tnaicator Diagrams from Allen Engine.

Cylinder 20 ins. diam, 30 ins. stroke.

100 Revolutions per minute.

speed af Piston 600 feet per minuze.

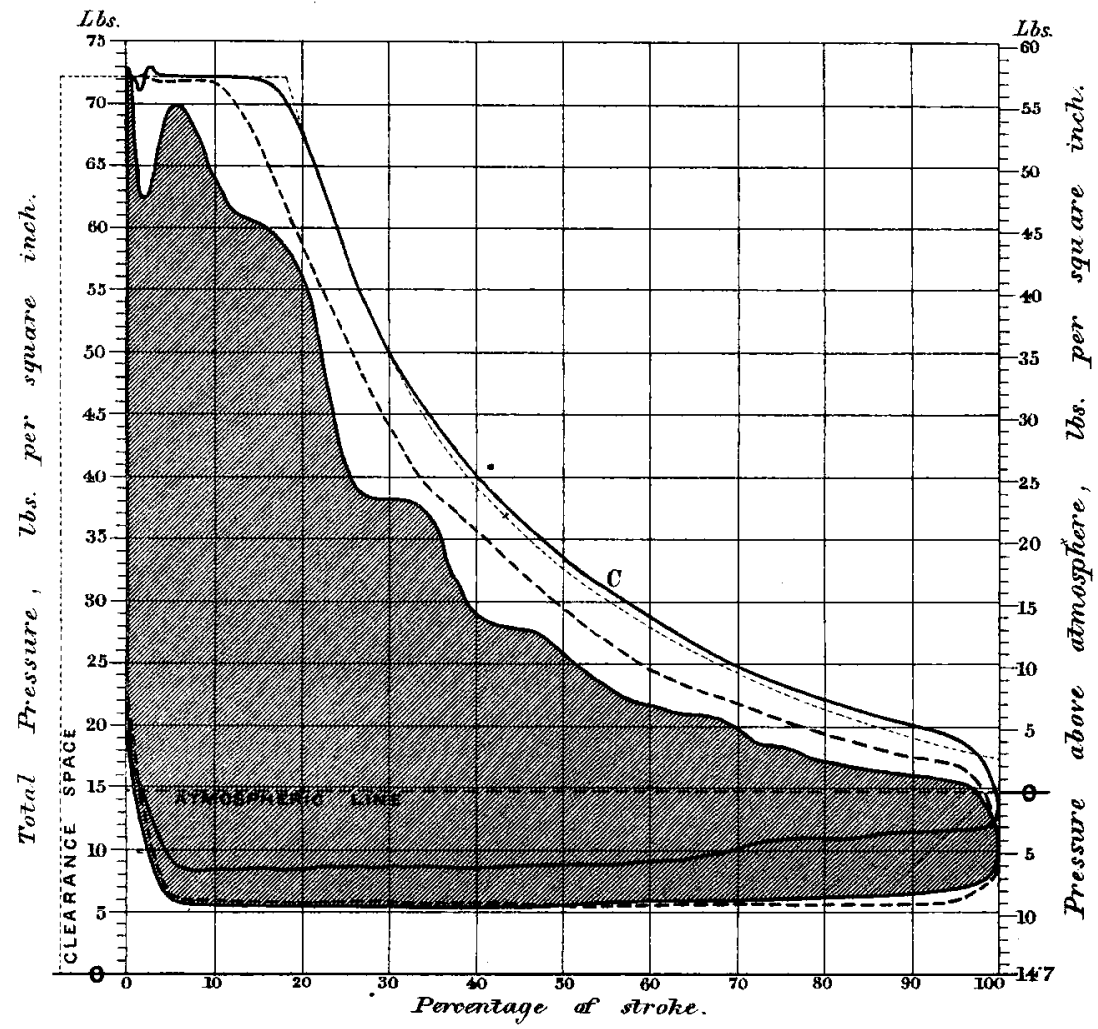

The fine dotted line shows the theoretical true expansion curve of the indicator diagram $\mathrm{C}$. 